\title{
Transforming growth factor- $\beta$ inhibits IQ motif containing guanosine triphosphatase activating protein 1 expression in lung fibroblasts via the nuclear factor- $\kappa B$ signaling pathway
}

\author{
CHUANYUE ZONG ${ }^{1 *}$, XIANLONG ZHANG ${ }^{2 *}$, YING XIE $^{1 *}$ and JIAWEN CHENG ${ }^{3}$ \\ ${ }^{1}$ Department of Anesthesiology, Huai'an Hospital Affiliated to Xuzhou Medical College and Huai'an Second People's Hospital, \\ Huai'an, Jiangsu 223002; ${ }^{2}$ Department of Anesthesiology, Huai'an First People's Hospital, Nanjing Medical University, Huai'an, \\ Jiangsu 223300; ${ }^{3}$ Department of Anesthesiology, The First People's Hospital of Suqian, Suqian, Jiangsu 223800, P.R. China
}

Received April 22,2014; Accepted January 26, 2015

DOI: $10.3892 / \mathrm{mmr} .2015 .3353$

\begin{abstract}
IQ motif containing guanosine triphosphatase activating protein 1 (IQGAP1) is associated with idiopathic pulmonary fibrogenesis (IPF); however, characterization of the expression of IQGAP1 in lung fibroblasts has remained elusive. The present study therefore evaluated IQGAP1 expression in mouse and human lung fibroblasts under fibrotic conditions via western blot analysis. It was revealed that IQGAP1 expression levels were significantly decreased in lung fibroblasts isolated from bleomycin-challenged mice than in those of control mice. Transforming growth factor- $\beta$ (TGF- $\beta$ ) induced differentiation, as well as decreased expression of IQGAP1 in WI-38 cells human lung fibroblasts. Furthermore, inhibition of nuclear factor (NF)- $\kappa B$ activation restored the TGF- $\beta$-induced inhibition of IQGAP1 expression in WI-38 cells. In lysophosphatidic acid (LPA)-challenged WI-38 cells, the expression of IQGAP1 was also decreased, while neutralized anti-TGF- $\beta$ antibody treatment restored the LPA-induced inhibition of IQGAP1 expression. These data indicated that TGF- $\beta$ inhibited IQGAP1 expression in lung fibroblasts via the NF- $\kappa \mathrm{B}$ signaling pathway, presenting a potential novel therapeutic target for the treatment of IPF.
\end{abstract}

\section{Introduction}

Idiopathic pulmonary fibrosis (IPF) is a fatal and highly destructive chronic interstitial lung disease, and the survival

Correspondence to: Dr Jiawen Cheng, Department of Anesthesiology, The First People's Hospital of Suqian, 120 Suzhi Road, Suqian, Jiangsu 223800, P.R. China

E-mail: chengjiawen2013@163.com

${ }^{*}$ Contributed equally

Key words: IQ motif containing guanosine triphosphatase activating protein 1 , lysophosphatidic acid, transforming growth factor- $\beta$, nuclear factor- $\kappa \mathrm{B}$ signaling, idiopathic pulmonary fibrosis time following initial diagnosis is just 2-5 years (1). To date, no effective therapeutic strategy for IPF, except lung transplantation, has been developed (2). Therefore, investigation into the pathological mechanisms underlying IPF is urgently required.

Amongst the factors associated with IPF, fibroblasts have been demonstrated to have a critical role (3). In IPF, fibroblast accumulation results in the irreversible destruction of lung architecture, and the differentiation of fibroblasts induces deposition of extracellular matrix (ECM) proteins, as well as $\alpha$-smooth muscle actin ( $\alpha$-SMA), under fibrotic conditions (3). The elucidation of fibroblast function is therefore critical for understanding the molecular mechanisms underlying IPF. Accumulating evidence has demonstrated that transforming growth factor- $\beta$ (TGF- $\beta$ ), sphingosine-1-phosphate (S1P) and lysophosphatidic acid (LPA) are involved in IPF (3-8). Recent studies indicated that TGF- $\beta$, LPA and S1P induced differentiation of lung fibroblasts, which was followed by increased expression of ECM proteins, such as fibronectin (FN) (4-6,9). Notably, during LPA stimulation of lung fibroblasts, TGF- $\beta$ expression increased via LPA receptor type 2-dependent pathways (5). However, the crosstalk between the LPA and TGF- $\beta$ signaling pathways in lung fibroblast differentiation has remained elusive.

IQ motif containing guanosine triphosphatase activating protein 1 (IQGAP1), an effector of CDC42, is a multidomain molecule implicated in the modulation of cell architecture and the regulation of exocytosis in multiple types of human cancer (10). In particular, IQGAP1 has been demonstrated to be expressed and localized in human cancer tissues (11-14). Recently, IQGAP1 was found to suppress TGF- $\beta /$ TGF- $\beta$ receptor 2 (RII)-dependent myofibroblastic differentiation in the tumor microenvironment (15). An in vitro study also indicated that IQGAP1 knockdown increased T $\beta$ RII stability, and TGF- $\beta 1$ induced the transdifferentiation of pericytes to myofibroblasts (15). Amongst patients with interstitial lung disease (ILD), the expression of IQGAP1 is increased in the lung fibroblasts of scleroderma patients with ILD, and IQGAP1 has crucial functions in the regulation of endothelial and epithelial cell migration (16). However, identification of IQGAP1 expression in pulmonary fibroblasts during fibrogenesis has remained elusive. 
The present study therefore examined the expression of IQGAP1 in mouse and human fibroblasts, in order to characterize its expression under fibrotic conditions.

\section{Materials and methods}

Reagents and kits. Bleomycin sulfate (BLM) was purchased from Hospira Inc. (Lakeforest, IL, USA). LPA (18:1) was obtained from Avanti Polar Lipids (Alabaster, AL, USA). Human TGF- $\beta 1$ protein was obtained from PeproTech, Inc. (Rocky Hill, NJ, USA). Cell lysis buffer was from Cell Signaling Technology Inc. (Danvers, MA, USA). Horseradish peroxidase-linked goat anti-mouse immunoglobulin G (IgG) (cat. no. 170-6516) and goat anti-rabbit IgG (cat. no. 170-6515) secondary antibodies were purchased from Bio-Rad Laboratories, Inc. (Hercules, CA, USA). The control chicken anti-goat polyclonal IgG (cat. no. BAF019) and neutralizing chicken polyclonal anti-TGF- $\beta 1$ (cat. no. AF-101-NA) antibodies were obtained from R\&D Systems (Minneapolis, MN, USA). Rabbit polyclonal anti-FN (cat. no. sc-9068) and rabbit polyclonal anti-IQGAP1 (cat. no. sc-10792) antibodies were obtained from Santa Cruz Biotechnology, Inc. (Dallas, TX, USA). Mouse monoclonal anti- $\alpha$-smooth muscle actin ( $\alpha$-SMA; cat. no. A2547), mouse monoclonal anti- $\beta$-actin (cat. no. A5316) and anti-Bay 11-7082 (NF- $\mathrm{B}$ inhibitor; cat. no. B5556) antibodies were obtained from Sigma-Aldrich (St. Louis, MO, USA).

Mouse model of IPF. The experimental model of pulmonary fibrosis was designed as described previously $(5,6)$. C57/BL6 mice (50 male mice, 8 weeks-old, weighing $\sim 25 \mathrm{~g}$ ) purchased from Jackson Laboratory (Bar Harbor, ME, USA) underwent bleomycin-induced fibrosis. The mice were housed under a controlled 12:12 h light:dark cycle. The air temperature was maintained at $22^{\circ} \mathrm{C}$ and the mice were given ad libitum access to food and water. The mice were anesthetized with a $3 \mathrm{ml} / \mathrm{kg}$ mixture of $25 \mathrm{mg} / \mathrm{kg}$ of ketamine in $2.5 \mathrm{ml}$ of xylazine (NCE Biomedical Co. Ltd. Wuhan, Hubei, China), followed by treatment with saline or bleomycin sulfate $(1.5 \mathrm{U} / \mathrm{kg}$ of body weight, $\sim 0.03$ U/animal) in saline by intratracheal injection in a total volume of $50 \mu \mathrm{l}$. A total of 21 days after bleomycin administration 34 mice were sacrificed using $\mathrm{CO}_{2}$ euthanasia, and the lungs were harvested for isolation of lung fibroblasts. All animal protocols conformed to the standards of Xuzhou Medical College (Huaian, China) and were in accordance with Chinese animal operation regulations. The present study was approved by the Ethics Committee of Xuzhou Medical College.

Isolation of mouse primary fibroblasts and lung fibroblast culture. Mouse lung fibroblasts were isolated as described previously $(5,6)$. The WI-38 human lung fibroblast cell line was purchased from the American Type Culture Collection (Manassas, VA, USA). Mouse primary lung fibroblasts and WI-38 cells were cultured in six-well dishes using Dulbecco's modified Eagle's medium (Life Technologies, Carlsbad, CA, USA), supplemented with $10 \%$ fetal bovine serum (Life Technologies).

Neutralizing antibodies or $N F-\kappa B$ inhibitor treatment. WI-38 cells ( $\sim 90 \%$ confluence) were serum-starved for $24 \mathrm{~h}$, and subsequently treated with neutralized control $\mathrm{IgG}$ or
anti-TGF- $\beta$ antibody $(5 \mu \mathrm{g} / \mathrm{ml})$ for $1 \mathrm{~h}$. The starved WI-38 cells were then incubated with $10 \mu \mathrm{M} \mathrm{NF- \kappa B}$ inhibitor (Bay 11-7082) for $1 \mathrm{~h}$. Subsequently, the fibroblasts were treated with TGF- $\beta(5 \mathrm{ng} / \mathrm{ml})$ or LPA $(10 \mu \mathrm{M})$ for $48 \mathrm{~h}$. Finally, the cells were lysed with cell lysis buffer (cat. no. 9803S; Cell Signaling Technology Inc.) and western blot analysis was used to evaluate protein expression.

Western blot analysis. Western blot analysis was used to evaluate protein expression and was performed as described previously (5). Cell lysates (20-30 $\mu \mathrm{g}$ protein, $1.5 \mathrm{mg} / \mathrm{ml}$ ) were cleared by centrifugation at $10,000 \mathrm{x} \mathrm{g}$ for $10 \mathrm{~min}$ and boiled with Laemmli sample buffer (Sigma-Aldrich) for $5 \mathrm{~min}$. The protein was assayed using the Pierce ${ }^{\mathrm{TM}}$ Bicinchoninic Acid Protein Assay kit (Thermo Fisher Scientific, Inc., Rockford, IL, USA). The cell lysates were separated by $10 \%$ or $4-20 \%$ SDS-PAGE and transferred to polyvinylidene fluoride (PVDF) membranes (Bio-Rad Laboratories, Inc.). The membranes were then blocked with tris-buffered saline-Tween (TBST) containing 5\% bovine serum albumin (Bio-Rad Laboratories, Inc.), prior to incubation with the primary antibodies (1:2,000 dilutions) overnight at $4^{\circ} \mathrm{C}$. Subsequently, the PVDF membranes were washed with TBST buffer and incubated with secondary antibodies for $2 \mathrm{~h}$ at room temperature (1:2,000 dilutions). All primary and secondary antibodies used were described in the reagents and kits section. The blots were visualized using an enhanced chemiluminescence kit (cat. no. ab65623; Abcam, Cambridge, MA, USA). The integrated density of pixels on each membrane was quantified and normalized to actin using Image Quant software version 5.2 (Molecular Dynamics, Sunnyvale, CA, USA).

Immunofluorescence microscopy. Immunofluorescence microscopy was used to examine protein expression as described previously (5). Briefly, mouse lung fibroblasts were incubated with the primary antibodies at a 1:200 dilution in blocking buffer (Bio-Rad Laboratories, Inc.) for $1 \mathrm{~h}$ at room temperature. Subsequently, the cells were incubated with the secondary antibodies with Alexa Fluor (Life Technologies) at a 1:200 dilutions in blocking buffer for $1 \mathrm{~h}$ at room temperature. Slides were examined under a Nikon Eclipse TE 2000-S fluorescence microscope (Nikon Corp., Tokyo, Japan) and recorded with a $60 \mathrm{X}$ oil immersion objective lens.

Statistical analysis. The data from at least three independent sets of experiments are displayed as the mean \pm standard error of the mean. For data analysis, one-way analysis of variance or a two-tailed Student's t-test in SPSS version 22.0 (IBM, Armonk, NY, USA) was used. P<0.05 was considered to indicate a statistically significant difference between values $(17,18)$.

\section{Results}

IQGAPI expression is decreased in the lung fibroblasts of BLM-treated mice. As shown in Fig. 1A-C, the expression of $\alpha$-SMA and FN was markedly higher in lung fibroblasts isolated from BLM-challenged mice than that in the fibroblasts of mice without BLM-challenge. However, the 
A

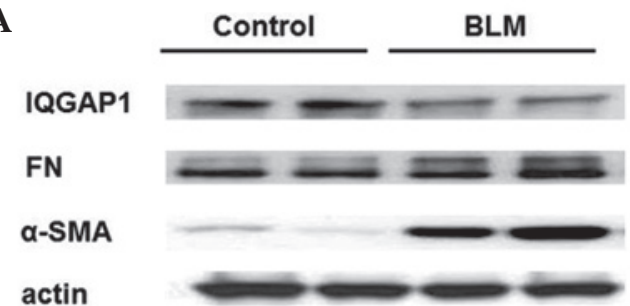

C

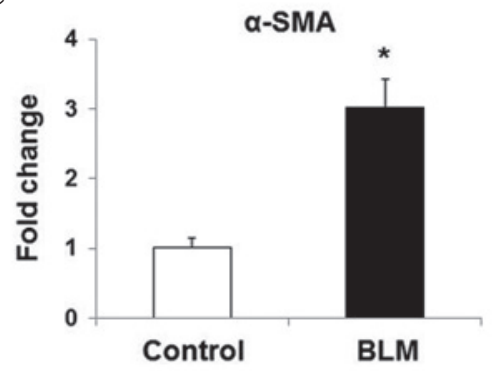

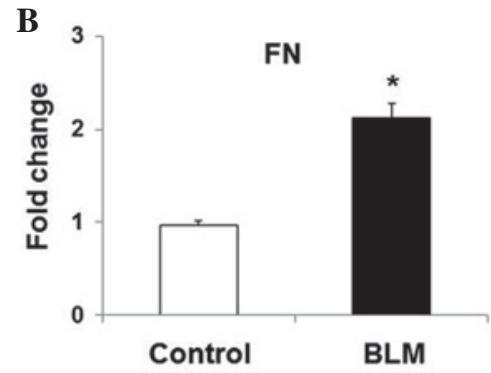

D

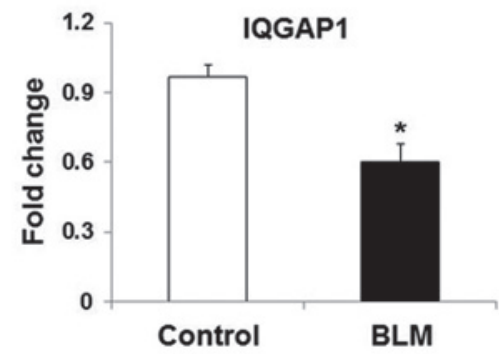

$\mathbf{E}$

IQGAP 1

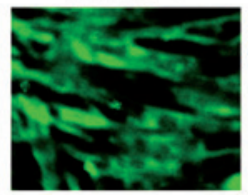

PBS

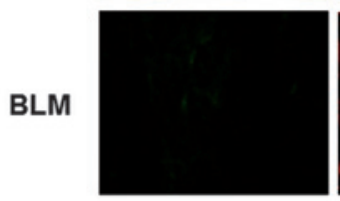

a-SMA
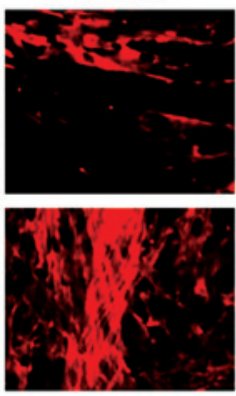

DAPI
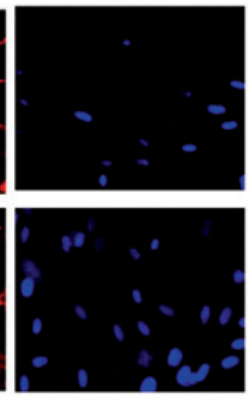

Merge
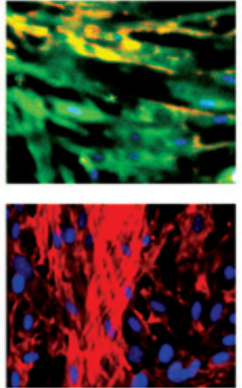

Figure 1. $\alpha$-SMA, FN and IQGAP1 expression in mouse lung fibroblasts. (A) Representative western blot of the expression of FN, $\alpha$-SMA, IQGAP1 and actin in mouse lung fibroblasts. Quantification of the expression of (B) FN, (C) $\alpha$-SMA and (D) IQGAP1. Values are presented as the mean \pm standard error of the mean. ${ }^{*}<0.05$, vs. fibroblasts from mice without BLM challenge (control). (E) Immunofloresent staining of IQGAP1 and $\alpha$-SMA in mouse lung fibroblasts (magnification, x60), $\alpha$-SMA (red), IQGAP1 (green) and DAPI (blue). $\alpha$-SMA, $\alpha$-smooth muscle actin; FN, fibronectin; IQGAP1, IQ motif containing guanosine triphosphatase activating protein 1; BLM, bleomycin sulfate; PBS, phophate-buffered saline.

IQGAP1 expression levels were significantly lower in lung fibroblasts from BLM-challenged mice than in those from control mice (Fig. 1A and D). Immunofluorescent staining also indicated that the expression levels of IQGAP1 were lower in lung fibroblasts from BLM-challenged mice, than in those of the control mice (Fig. 1E).

TGF- $\beta$ downregulates the expression of IQGAPI in WI-38 human lung fibroblast cells. In order to determine whether the expression of IQGAP1 was associated with lung fibroblast activation and differentiation, TGF- $\beta$-induced IQGAP1 expression was examined in WI-38 cells. TGF- $\beta$ treatment ( $5 \mathrm{ng} / \mathrm{ml}, 48 \mathrm{~h}$ ) induced fibroblast differentiation, characterized by the enhanced expression of $\alpha$-SMA and FN (Fig. 2A-C), as well as significantly inhibiting the expression of IQGAP1 in WI-38 cells (Fig. 2A and D).

TGF- $\beta$ inhibits IQGAPI expression via the NF- $\kappa B$ pathway. To further examine the potential mechanisms underlying the TGF- $\beta$-induced inhibition of IQGAP1 expression in lung fibroblasts, WI-38 cells were treated with $\mathrm{NF}-\kappa \mathrm{B}$ inhibitor (Bay $11-7082 ; 10 \mu \mathrm{M}$ ) for $1 \mathrm{~h}$ prior to TGF- $\beta$-challenge. As indicated in Fig. 3, TGF- $\beta$ challenge induced upregulation of the expression of $\alpha$-SMA and $F N$, and $N F-\kappa B$ inhibitor treatment markedly attenuated the effects of TGF- $\beta$. Notably, the inhibitory role of TGF- $\beta$ in IQGAP1 expression in WI-38 cells was also significantly restored following the inhibition of $\mathrm{NF}-\kappa \mathrm{B}$ pathways (Fig. 3A and D). Therefore, these results indicated that TGF- $\beta$ inhibited IQGAP1 expression via the $\mathrm{NF}-\kappa \mathrm{B}$ signaling pathways.

LPA downregulates the expression of IQGAP in WI38 cells via TGF- $\beta$-dependent pathways. LPA has been demonstrated to be involved in lung fibrogenesis, particularly by inducing the activation and recruitment of fibroblasts $(4,5)$. Recently, LPA was revealed to enhance TGF- $\beta$ expression levels in human lung fibroblasts, potentially via LPA receptor type 2-associated pathways (5). The present study therefore evaluated the effects of LPA on IQGAP1 expression in WI-38 cells. LPA treatment was demonstrated to induce an increase 
A

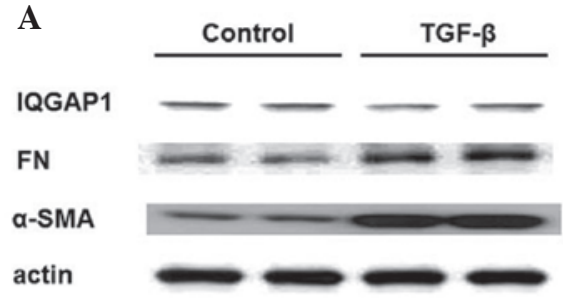

C

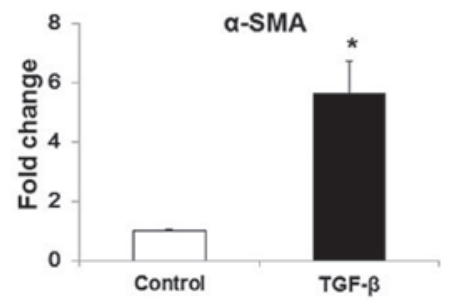

B

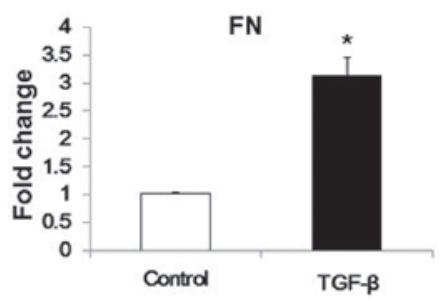

D

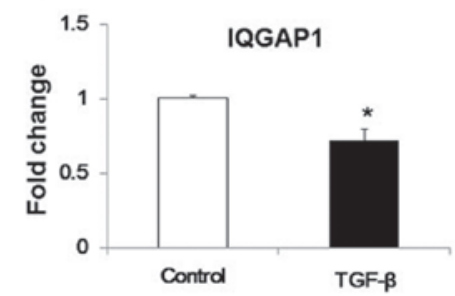

Figure 2. TGF- $\beta$ effects on the expression of FN, $\alpha$-SMA and IQGAP1 in human lung fibroblasts. Human lung fibroblasts were treated with TGF- $\beta(5 \mathrm{ng} / \mathrm{ml})$ prior to western blot analysis. (A) Representative western blot of protein expression of $\alpha$-SMA, FN, actin and IQGAP1. Quantification of (B) FN, (C) $\alpha$-SMA and (D) IQGAP1 expression in TGF- $\beta$-challenged WI-38 cells. Values are expressed as the mean \pm standard error of the mean of three independent experiments. "P<0.05, vs. cells without TGF- $\beta$ challenge (control). TGF- $\beta$, transforming growth factor- $\beta$; $\alpha$-SMA, $\alpha$-smooth muscle actin; FN, fibronectin; IQGAP1, IQ motif containing guanosine triphosphatase activating protein 1 .

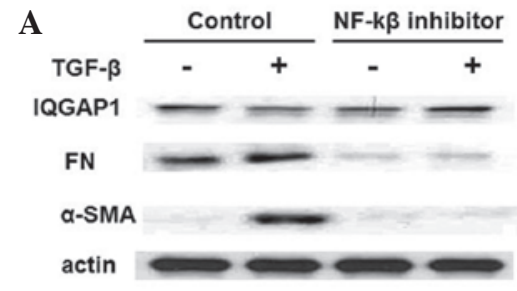

$\mathbf{C}$

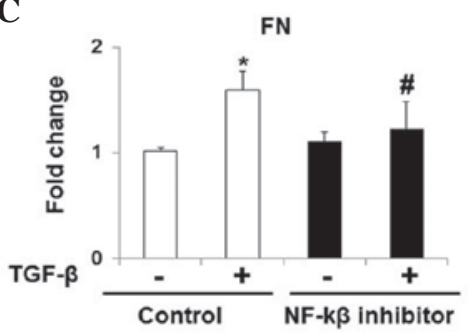

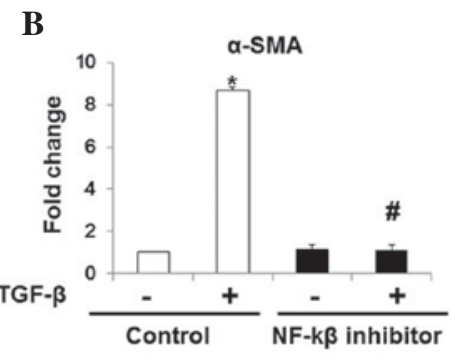

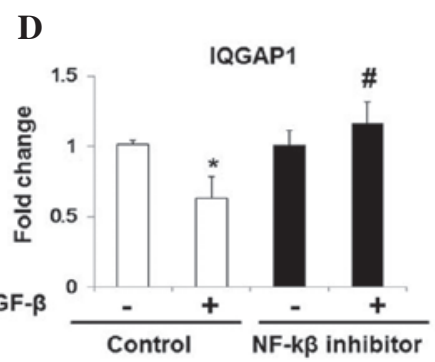

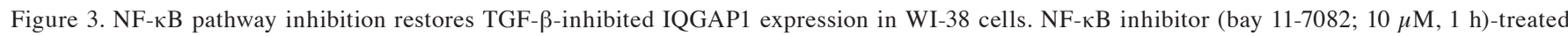
WI-38 cells were challenged with TGF- $\beta(5 \mathrm{ng} / \mathrm{ml}, 48 \mathrm{~h}$ ), and protein expression levels were analyzed by western blot analysis. (A) Representative western blot of protein expression of $\alpha$-SMA, FN, actin and IQGAP1. Quantification of the protein expression levels of (B) FN, (C) $\alpha$-SMA and (D) IQGAP1 in WI-38 cells with or without TGF- $\beta$-challenge. Values are expressed as the mean \pm standard error of the mean. ${ }^{\prime} \mathrm{P}<0.05$, vs. WI- 38 cells without TGF- $\beta$ treatment; ${ }^{"} \mathrm{P}<0.05$,

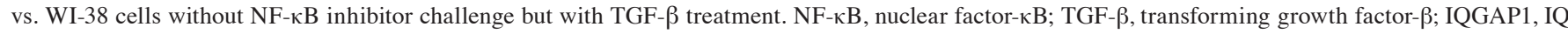
motif containing guanosine triphosphatase activating protein $1 ; \alpha$-SMA, $\alpha$-smooth muscle actin; FN, fibronectin.

in $\alpha$-SMA and FN expression levels, and inhibit IQGAP1 expression in WI38cells (Fig. 4). In order to determine whether LPA-induced TGF- $\beta$ secretion was associated with the effects of LPA on IQGAP1 expression in WI-38 cells, cells were pre-incubated with chicken anti-TGF- $\beta$ antibody $(5 \mathrm{ng} / \mathrm{ml})$ for $1 \mathrm{~h}$. As shown in Fig. 5, anti-TGF- $\beta 1$ antibody markedly inhibited LPA-induced fibroblast differentiation, and significantly increased IQGAP1 expression under LPA treatment (Fig. 5).

\section{Discussion}

IPF is a chronic lung disease, for which there is currently a lack of effective treatment. Lung transplantation is the only effective clinical therapeutic approach for IPF $(1,2,19)$. Inflammatory cells, epithelial cells and fibroblasts are involved in pulmonary fibrogenesis, and the expression of various bioactive ligands are altered during the fibrogenesis $(3,20)$. TGF- $\beta$ and LPA have been demonstrated to be the key factors underlying 
A

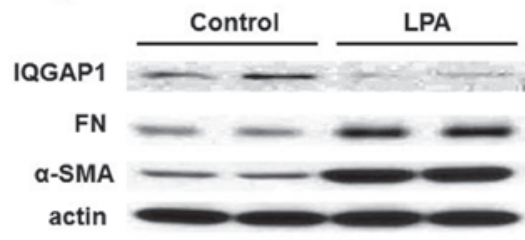

C

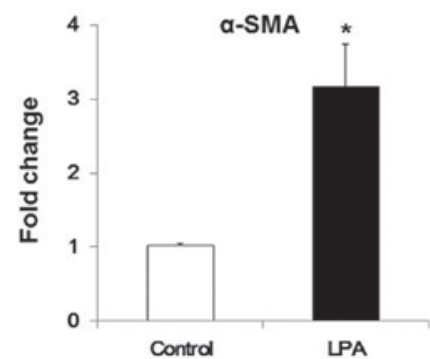

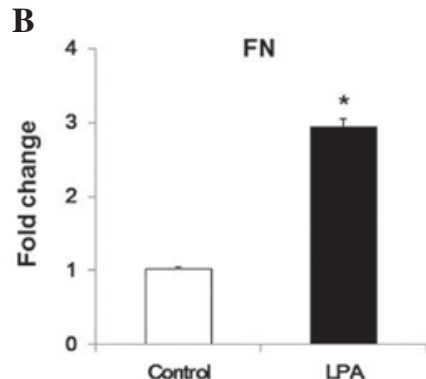

D

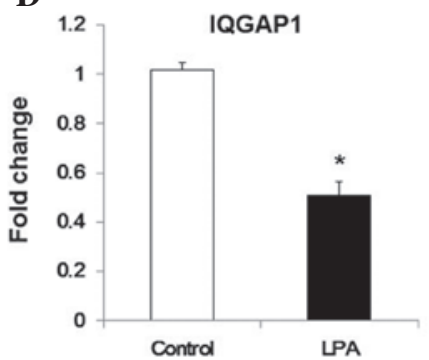

Figure 4. LPA induces differentiation and inhibition of IQGAP expression in WI-38 cells. (A) Representative western blot. Quantification of the expression of (B) FN, (C) $\alpha$-SMA and (D) IQGAP1 induced by LPA $(10 \mu \mathrm{M}, 48 \mathrm{~h})$ in human lung fibroblasts. Values are expressed as the mean \pm standard error of the mean of three independent experiments. $\mathrm{P}<0.05$, vs. cells without LPA treatment. LPA, lysophosphatidic acid; IQGAP1, IQ motif containing guanosine triphosphatase activating protein $1 ; \mathrm{FN}$, fibronectin; $\alpha$-SMA, $\alpha$-smooth muscle actin.

A

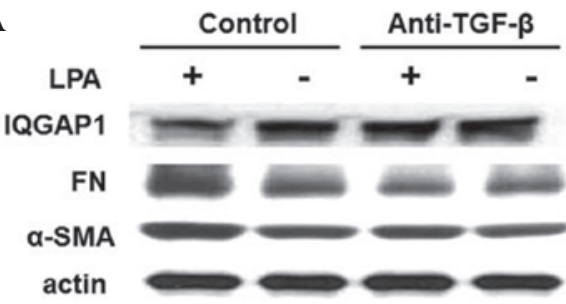

C

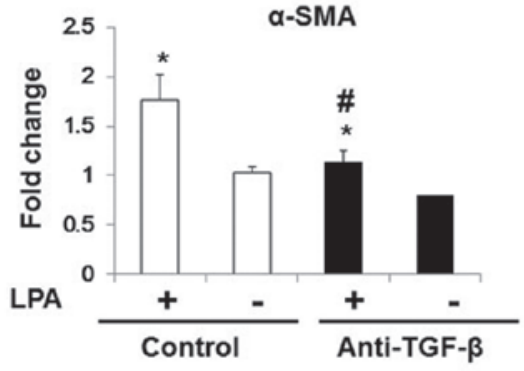

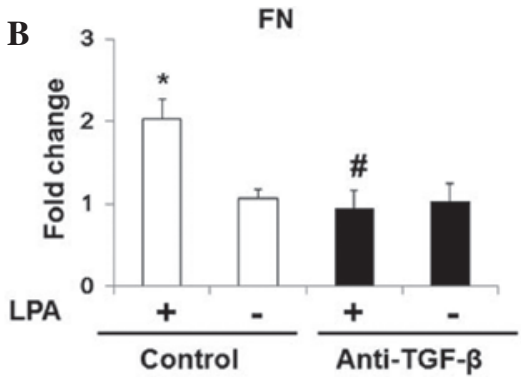

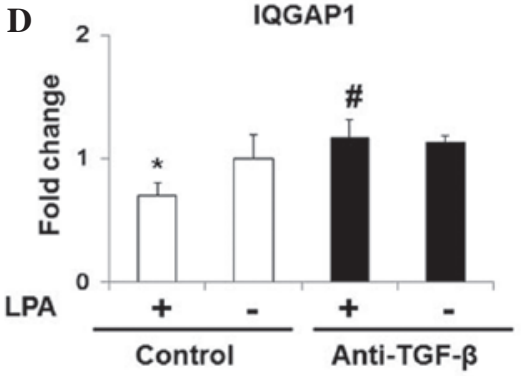

Figure 5. Anti-TGF- $\beta$ antibody restores LPA induced inhibition of IQGAP1 expression in WI-38 cells. Starved WI-38 cells were treated with control immunoglobulin $\mathrm{G}$ and anti-TGF- $\beta$ antibody $(5 \mu \mathrm{g} / \mathrm{ml})$ for $1 \mathrm{~h}$, and further challenged with 18:1 LPA $(0,10,20$ or $30 \mu \mathrm{M})$ for $48 \mathrm{~h}$. (A) Representative western blot for protein expression of $\alpha$-SMA, FN, actin and IQGAP1. Quantification of the protein expression of (B) FN, (C) $\alpha$-SMA and (D) IQGAP1 in WI-38 cells. Values are expressed as the mean \pm standard error of the mean. ${ }^{*} \mathrm{P}<0.05$, vs. WI-38 cells with control antibody but without LPA challenge; ${ }^{*} \mathrm{P}<0.05$, vs. LPA-challenged WI-38 cells pre-treated with control antibody. TGF- $\beta$, transforming growth factor- $\beta$; LPA, lysophosphatidic acid; IQGAP1, IQ motif containing guanosine triphosphatase activating protein $1 ; \mathrm{FN}$, fibronectin; $\alpha$-SMA, $\alpha$-smooth muscle actin.

fibrogenesis, and to regulate the activation and differentiation of fibroblasts $(4,5,6)$. Therefore, investigation into the effects of TGF- $\beta$ and LPA on fibrogenesis are essential for the elucidation of the pathological mechanisms underlying IPF.

IQGAP1, which is one of three IQGAP homologs, is expressed in all human organs $(21,22)$. Increasing evidence has indicated that IQGAP1 regulates cell migration and adhesion, extracellular signals and cytokinesis in multiple types of cell $(23,24)$. An oncological study revealed that IQGAP1 expression and localization are frequently altered in tumor tissues, and its expression is correlated with cancer progression (10). According to western blot and quantitative 
polymerase chain reaction analyses, the expression of IQGAP1 is also increased in various types of cancer cell $(23,24)$. Detailed analysis of this effect indicated that IQGAP1 was associated with the $\beta$-catenin and extracellular signal-regulated kinase (ERK) signaling pathways. It also demonstrated that IQGAP1 also binds with mitogen-activated protein kinase kinase, B-Raf and ERK to regulate their sequential signaling cascades, resulting in tumorigenesis in humans and experimental animals $(25,26)$. Investigation in liver cancer indicated that IQGAP1 inhibited the TGF- $\beta / T \beta R$ RI/ myofibroblast differentiation signaling pathway and blocked tumor growth in the tumor tissues. These effects are associated with the binding of IQGAP1 to T $\beta R I I$, inducing the ubiquitination and degradation of hepatic stellate cells (15). A recent study indicated that PDGF stimulation rapidly promotes the association of IQGAP1 with PDGF receptor- $\beta$ (PDGFR), and overexpression of IQGAP1 enhances PDGFR autophosphorylation (27). Thus suggesting that through interaction with PDGFR and focal adhesions signaling proteins, IQGAP1 promotes activation of PDGFR in focal adhesions, and contributes to vascular smooth muscle cell migration and neointimal formation after injury (27). In addition, IQGAP1 increases bronchial epithelial cell proliferation and wound closure via the phosphorylation of IQGAP1 (28). However, to the best of our knowledge, the expression of IQGAP1 during pulmonary fibrosis has not previously been investigated, and the potential roles of IQGAP1 in fibroblasts have remained elusive. In the present study, the expression of IQGAP1 was evaluated in human and mouse lung fibroblasts during pulmonary fibrosis. The results suggested that the expression of IQGAP1 was markedly decreased in pulmonary fibroblasts under fibrotic conditions. These studies therefore suggested that IQGAP1 expression was correlated with the differentiation of lung fibroblasts and lung fibrosis.

Various bioactive lipid compounds have been implicated in inflammation and pulmonary fibrosis $(5,6,17,18,29-33)$. In particular, S1P and LPA induce fibroblast recruitment and differentiation during pulmonary fibrogenesis (4-6). LPA also induces the expression of TGF- $\beta$ in fibroblasts from human lung tissue in a dose-dependent manner, through activation of LPA receptor type 2 (5). The present study indicated that LPA also inhibited IQGAP1 expression via TGF- $\beta$ secretion, which suggested that there was crosstalk between the LPA and TGF- $\beta$ signaling pathways in the regulation of IQGAP1 expression in lung fibroblasts during IPF.

In conclusion, the results of the present study revealed that TGF- $\beta$ inhibited the expression of IQGAP1 via activation of the NF- $\kappa \mathrm{B}$ signaling pathway, and suggested that targeting IQGAP1 to inhibit pulmonary fibrosis may present potential novel therapeutic strategies for the treatment of IPF.

\section{References}

1. Huang LS and Natarajan V: Sphingolipids in pulmonary fibrosis Adv Biol Regul 57C: 55-63, 2015.

2. Marks JH: Update in pulmonary medicine. Adolesc Med State Art Rev 24: 307-329, 2013.

3. Wynn TA: Integrating mechanisms of pulmonary fibrosis. J Exp Med 208: 1339-1350, 2011

4. Tager AM, LaCamera P, Shea BS, et al: The lysophosphatidic acid receptor LPA (1) links pulmonary fibrosis to lung injury by mediating fibroblast recruitment and vascular leak. Nat Med 14: $45-54,2008$
5. Huang LS, Fu P, Patel P, Harijith A, Sun T, Zhao Y, Garcia JG, Chun J and Natarajan V: Lysophosphatidic acid receptor 2 deficiency confers protection against bleomycin-induced lung injury and fibrosis in mice. Am J Respir Cell Mol Biol 49: 912-922, 2013.

6. Huang LS, Berdyshev E, Mathew B, et al: Targeting sphingosine kinase 1 attenuates bleomycin-induced pulmonary fibrosis. FASEB J 27: 1749-1760, 2013.

7. Natarajan V,DudekSM,JacobsonJR,etal:Sphingosine-1-phosphate, FTY720 and sphingosine-1-phosphate receptors in the pathobiology of acute lung injury. Am J Respir Cell Mol Biol 49: 6-17, 2013.

8. Bartram $\mathrm{U}$ and Speer $\mathrm{CP}$ : The role of transforming growth factor beta in lung development and disease. Chest 125: 754-765, 2004.

9. Rancoule C, Pradere JP, Gonzalez J, Klein J, Valet P, Bascands JL, Schanstra JP and Saulnier-Blache JS: Lysophosphatidic acid-1-receptor targeting agents for fibrosis. Expert Opin Investig Drugs 20: 657-667, 2011.

10. Johnson M, Sharma M and Henderson BR: IQGAP1 regulation and roles in cancer. Cell Signall 21: 1471-1478, 2009.

11. Nabeshima K, Shimao $Y$, Inoue $T$ and Koono $M$ : Immunohistochemical analysis of IQGAP1 expression in human colorectal carcinomas: its overexpression in carcinomas and association with invasion fronts. Cancer Lett 176: 101-109, 2002.

12. Clark EA, Golub TR, Lander ES and Hynes RO: Genomic analysis of metastasis reveals an essential role for RhoC. Nature 406: 532-535, 2000.

13. Brown MD and Sacks DB: IQGAP1 in cellular signaling: bridging the GAP. Trends Cell Biol 16: 242-249, 2006.

14. Takemoto H, Doki Y, Shiozaki H, Imamura H, Utsunomiya $T$, Miyata $\mathrm{H}$, Yano $\mathrm{M}$, Inoue $\mathrm{M}$, Fujiwara $\mathrm{Y}$ and Monden $\mathrm{M}$ : Localization of IQGAP1 is inversely correlated with intercellular adhesion mediated by e-cadherin in gastric cancers. Int J Cancer 91: 783-788, 2001.

15. Liu C, Billadeau DD, Abdelhakim H, Leof E, Kaibuchi K, Bernabeu C, Bloom GS, Yang L, Boardman L, Shah VH and Kang N: IQGAP1 suppresses TbetaRII-mediated myofibroblastic activation and metastatic growth in liver. J Clin Invest 123: 1138-1156, 2013.

16. Bogatkevich GS, Ludwicka-Bradley A, Singleton CB, Bethard JR and Silver RM: Proteomic analysis of CTGF-activated lung fibroblasts: identification of IQGAP1 as a key player in lung fibroblast migration. Am J Physiol Lung Cell Mol Physiol 295: L603-L611, 2008.

17. HuangLS,HungND, SokDEandKimMR:Lysophosphatidylcholine containing docosahexaenoic acid at the sn-1 position is anti-inflammatory. Lipids 45: 225-236, 2010.

18. Huang LS, Kang JS, Kim MR and Sok DE: Oxygenation of arachidonoyl lysophospholipids by lipoxygenases from soybean, porcine leukocyte, or rabbit reticulocyte. J Agric Food Chem 56: 1224-1232, 2008.

19. King TE: Update in pulmonary medicine. Ann Intern Med 129: 806-812, 1998.

20. Wolters PJ, Collard HR and Jones KD: Pathogenesis of idiopathic pulmonary fibrosis. Annu Rev Pathol 9: 157-79, 2014.

21. Weissbach L, Settleman J, Kalady MF, Snijders AJ, Murthy AE, Yan YX and Bernards A: Identification of a human rasGAP-related protein containing calmodulin-binding motifs. J Biol Chem 269: 20517-20521, 1994.

22. Wang S, Watanabe T, Noritake J, Fukata M, Yoshimura T, Itoh N, Harada T, Nakagawa M, Matsuura Y, Arimura N and Kaibuchi K: IQGAP3, a novel effector of Rac1 and Cdc42, regulates neurite outgrowth. J Cell Sci 120: 567-577, 2007.

23. Jadeski L, Mataraza JM, Jeong HW, Li Z and Sacks DB: IQGAP1 stimulates proliferation and enhances tumorigenesis of human breast epithelial cells. J Biol Chem 283: 1008-1017, 2008.

24. Dong PX, Jia N, Xu ZJ, Liu YT, Li DJ and Feng YJ: Silencing of IQGAP1 by shRNA inhibits the invasion of ovarian carcinoma HO-8910PM cells in vitro. J Exp Clin Cancer Res: CR 27: 77, 2008.

25. Ren JG, Li Z and Sacks DB: IQGAP1 modulates activation of B-Raf. Proc Nat Acad Sci USA 104: 10465-10469, 2007.

26. Roy M, Li Z and Sacks DB: IQGAP1 is a scaffold for mitogen-activated protein kinase signaling. Mol Cell Biol 25: 7940-7952, 2005

27. Kohno T, Urao N, Ashino T, Sudhahar V, Inomata H, Yamaoka-Tojo M, McKinney RD, Fukai T and Ushio-Fukai M: IQGAP1 links PDGF receptor-beta signal to focal adhesions involved in vascular smooth muscle cell migration: role in neointimal formation after vascular injury. Am J Physiol Cell Physiol 305: C591-C600, 2013. 
28. Wang Y, Wang M, Wang F, Zhu M, Ma Y, Wang X and Wu R IQGAP1 promotes cell proliferation and is involved in a phosphorylation-dependent manner in wound closure of bronchial epithelial cells. Int J Mol Med 22: 79-87, 2008.

29. Huang LS, Kim MR and Sok DE: Linoleoyl lysophosphatidylcholine is an efficient substrate for soybean lipoxygenase-1. Arch Biochem Biophys 455: 119-126, 2006.

30. Huang LS, Kim MR and Sok DE: Oxygenation of 1-docosahexaenoyl lysophosphatidylcholine by lipoxygenases; conjugated hydroperoxydiene and dihydroxytriene derivatives. Lipids 42: 981-990, 2007.
31. Huang LS, Kim MR and Sok DE: Regulation of lipoxygenase activity by polyunsaturated lysophosphatidylcholines or their oxygenation derivatives. J Agric Food Chem 56: 7808-7814, 2008.

32. Huang LS, Kim MR and Sok DE: Enzymatic reduction of polyunsaturated lysophosphatidylcholine hydroperoxides by glutathione peroxidase-1. Eur J Lipid Sci Tech 111: 584-592, 2009.

33. Huang LS, Mathew B, Li H, et al: The mitochondrial cardiolipin remodeling enzyme lysocardiolipin acyltransferase is a novel target in pulmonary fibrosis. Am J Respir Crit Care Med 189: $1402-1415,2014$ 